$63^{\text {ème }}$ Congrès de la SFCO, 02016 (2015)

DOI:10.1051/sfco/20156302016

(C) Owned by the authors, published by EDP Sciences, 2015

\title{
COMMUNICATION
}

\section{Fibrome ossifiant ou dysplasie fibreuse : difficulté de diagnostic clinique, radiologique et histologique}

\author{
Nabih O, Medaghri Alaoui O, Benyahya I \\ Faculté de médecine dentaire de Casablanca, Rue Abou Al Alaa Zahar Mers Sultan, Casablanca, Maroc
}

Le fibrome ossifiant et la dysplasie fibreuse constituent deux lésions fibro-osseuses. Ces lésions ont toutes en commun le remplacement du tissu osseux normal par un tissu composé de fibres de collagène et de fibroblastes, contenant une quantité variable de substance minéralisée (Hourani et al. 2014).

Ces deux lésions sont caractérisées par une croissance lente et asymptomatique et présentent dans de nombreux cas, des aspects histologiques comparables ce qui complique le diagnostic différentiel et constitue un vrai dilemme pour le praticien ainsi que pour l'anatomopathologiste (Shi et al. 2013).

Un cas clinique rapporté en est l'exemple, il a posé un problème de diagnostic différentiel entre le fibrome ossifiant et la dysplasie fibreuse, vu la similitude clinico-radio-histologique qui existe entre ces deux entités.

Il s'agit d'une femme de 28 ans qui s'est présentée pour une tuméfaction au niveau maxillaire. L'examen clinique révèle la présence d'une masse de $2 \mathrm{~cm}$ intéressant la crête édentée entre la 24 et la 27, s'étendant dans le sens vestibulo-palatin et jusqu'à la surface occlusale des dents adjacentes. Cette masse qui fait corps avec l'os alvéolaire, est recouverte d'une muqueuse d'aspect sain, et elle est ferme et indolore à la palpation. Le reste de l'examen est non contributif. L'orthopantomogramme montre une image mixte (radioclaire et radiopaque) constituée de proportion variable de zones ossifiées radiodenses, et de zones d'os néoformé de densité inférieure à l'os compact.

L'exérèse de la lésion est pratiquée sous anesthésie locale et la pièce opératoire est adressée à deux laboratoires différents. Ces deux laboratoires ont décrit le même tableau histopathologique mais avec deux conclusions distinctes, l'un a identifié un fibrome ossifiant et l'autre une dysplasie fibreuse.

Le diagnostic est alors posé en faveur de la dysplasie fibreuse en se basant sur les éléments cliniques, radiologiques et épidémiologiques.

Toutefois, il existe deux méthodes fiables et précises permettant de distinguer ces deux entités en cas de difficulté de diagnostic et qui sont l'étude immunohistochimique de l'ostéocalcine ainsi que la recherche de la mutation du gène qui code la sous unité $\alpha$ de la protéine G. L'ostéocalcine est retrouvée en abondance dans la dysplasie fibreuse et la mutation du gène qui code la sous unité $\alpha$ est incriminé dans son origine (Shi et al. 2013).

This is an Open Access article distributed under the terms of the Creative Commons Attribution License 4.0, which permits unrestricted use, distribution, and reproduction in any medium, provided the original work is properly cited. 
Le diagnostic est important dans le traitement et le pronostic de ces deux lésions car en cas de fibrome ossifiant, il est nécessaire de faire une énucléation complète et les récidives sont minimes. Alors que la dysplasie fibreuse doit être traitée selon le contexte clinique, la croissance de la dysplasie fibreuse a tendance à s’arrêter une fois la maturité du squelette est atteinte (Andrade et al. 2013).

Nom et adresse du conférencier

Ossama NABIH

Faculté de médecine dentaire de casablanca

Rue Abou Al Alaa Zahar Mers Sultan

Casablanca (Maroc)

ossama.nabih@gmail.com 